\title{
Transcriptome Analysis Reveals the Procyanidin Treatment-responsive Genes Involved in Regulating Procyanidin Accumulation during Banana Ripening and Senescence
}

\author{
Chao Zhou and Haide Zhang \\ College of Food Science and Engineering, Hainan University, Haikou 570228, China \\ Yixing Li, Fenfang Li, Jiao Chen, and Debao Yuan \\ Key Laboratory of Banana Genetic Improvement, Haikou Experimental Station, Chinese Academy of \\ Tropical Agricultural Sciences, Haikou 571101, China \\ Keqian Hong \\ Key Laboratory for Postharvest Physiology and Technology of Tropical Horticultural Products of \\ Hainan Province, South Subtropical Crop Research Institute, Chinese Academy of Tropical \\ Agricultural Sciences, Zhanjiang 524088, China
}

\begin{abstract}
AdDitional INDEX wORDs. biosynthetic enzymes, procyanidin biosynthesis, regulatory factors
ABstract. The mechanism regulating procyanidin (PA) accumulation in banana (Musa acuminata) fruit is not understood. During this study, the effects of PA treatment on the activities of banana PA biosynthetic enzymes and transcriptomic profiles were investigated. The results showed that $P A$ treatment delayed the decreases in leucoanthocyanidin reductase and anthocyanidin reductase activities, which affected the accumulation of PA. Furthermore, the peel samples of the control fruit and the PA-treated fruit on day 1 were selected for transcriptomic analysis. The results revealed that PA treatment induced 1086 differentially expressed genes. Twenty-one key genes, including those encoding biosynthetic enzymes and regulatory factors involved in PA biosynthesis, were validated using a quantitative real-time polymerase chain reaction. The results showed that these genes were upregulated by $P A$ treatment during banana storage. Taken together, our study improves current understanding of the mechanism underlying PA-regulated banana senescence and provide new clues for investigating specific gene functions.
\end{abstract}

Banana (Musa acuminata) is a commercially valuable climacteric fruit with ethylene peak. Once ripening is initiated, senescence is irreversible and rapid, as manifested by softening, peel spotting, and fungal decay, and leads to a short shelf (Yun et al., 2019). Therefore, knowledge of banana fruit ripening and senescence may assist in developing strategies that can improve the sensorial quality and reduce postharvest fruit losses. Previous studies have investigated the mechanisms involved in banana fruit ripening and senescence at physiological, biochemical, and molecular levels (Elitzur et al., 2016). However, considering the complexity of the ripening and senescence processes, most of the mechanisms regulating ripening and senescence in banana remain unidentified.

Polyphenols and flavonoids are the most abundant secondary metabolites that are widely distributed in the plant kingdom (Chibane et al., 2019). Furthermore, they can be applied safely

Received for publication 4 Nov. 2020. Accepted for publication 21 Dec. 2020. Published online 27 January 2021.

This work supported by the Hainan Natural Science Foundation of China (318QN288), Hainan Key R\&D Project (ZDYF2019208), and the Central Public-interest Scientific Institution Basal Research Fund for the Chinese Academy of Tropical Agricultural Sciences (1630092020004; 1630092020001; 1630092018008).

J.C. and D.Y. are the corresponding authors. E-mail: chenjiao52@163.com or yuandebao@163.com.

This is an open access article distributed under the CC BY-NC-ND license (https://creativecommons.org/licenses/by-nc-nd/4.0/). and conveniently; therefore, they are used as exogenous antioxidants for reducing oxidative stress. Reports have shown that the treatment of postharvest fruit with plant phenolic extracts, such as apple (Malus pumila) polyphenols (Zhang et al., 2015), tea (Camellia sinensis) polyphenols (Chen et al., 2014), and chlorogenic acid (Xi et al., 2017), could maintain the redox balance of fruit, delay senescence, and prolong the shelf life of fruit. As one of the most consumed polyphenols in the human diet, procyanidins (PAs), or condensed tannins, are derived from the condensation of the widely distributed flavan-3-ol in the plant (Yang et al., 2019). Until now, PAs have been reported to possess antioxidant, antimicrobial, antiviral, anti-inflammatory, and anticancer properties (Xiao et al., 2018). Furthermore, PAs, as natural food-borne preservatives, have been mainly used in meat and other food products alone or in combination with other technologies (Jing et al., 2019). However, the application of exogenous PA in fruit preservation is limited. Previously, we observed that the decrease in the PA content was delayed by exogenous PA treatment, thereby improving the antioxidant capacity of fruit tissue and delaying senescence of harvested banana fruit (Chen et al., 2019). Therefore, biosynthesis and accumulation of PA are important for delaying banana fruit senescence, although the relevant regulatory mechanism still warrants investigations.

The biosynthesis of PA is a part of the flavonoid pathway that has been well-characterized over the past several decades with the identification of numerous structural, regulatory, and 
transport-related genes (Yang et al., 2019). The enzyme genes, including phenylalanineammonia-lyase (PAL), cinnamic acid4-hydroxylase (C4H), chalconesynthase (CHS), chalconeisomerase $(\mathrm{CHI})$, flavanone3-hydroxylase $(\mathrm{F} 3 \mathrm{H})$, flavonoid $3^{\prime}$-hydroxylase $\left(\mathrm{F} 3{ }^{\prime} \mathrm{H}\right)$, dihydroflavonol4-reductase (DFR), anthocyanidin synthase (ANS), leucoanthocyanidin reductase (LAR), and anthocyanidin reductase (ANR), encode the corresponding structural enzymes responsible for the biochemical reactions of PA synthesis (Winkel-Shirley, 2001). In addition, numerous studies have demonstrated that three types of transcription factors (TFs) tryptophan-aspartate (WD)-repeat-containing proteins consisting of four or more copies of the WD repeat (WD40), basic helix-loop-helix (bHLH), and myeloblastosis (MYB), form MYB-bHLH-WD40 (MBW) ternary complexes to regulate the biosynthesis of anthocyanin and PA in all investigated plants (Deluc et al., 2006; Liu et al., 2018). For example, in Arabidopsis thaliana, four MBW complexes, PAP1(MYB75)-TT8/GL3-TTG1(WD40), PAP2(MYB90)TT8/GL3-TTG1, MYB113(PAP3)-TT8/GL3-TTG1, and PAP4(MYB114)-TT8/GL3-TTG1, have been shown to activate the expression of late anthocyanin biosynthetic genes, such as $A t D F R, A t A N S / L D O X$, and UF3GT (Shi and Xie, 2014). The MBW complex TT2 (MYB123)-TT8/GL3-TTG1 regulates the expression of PA biosynthetic genes AtDFR, AtANS/LDOX, and AtANR/BANYULS (BAN) (Xu et al., 2014). However, knowledge regarding the regulation of PA biosynthesis in banana fruit by structural and regulatory genes is currently limited.

In this study, the mechanism of PA treatment involved in in vivo PA accumulation in banana fruit during storage was investigated. The effects of PA treatment on activities of PA biosynthetic enzymes LAR and ANR during storage were assessed. Furthermore, PA-induced differentially expressed genes (DEGs) in banana fruit were analyzed using transcriptome analysis. Then, genes encoding the key biosynthetic enzymes and regulatory factors controlling PA biosynthesis were screened and validated during banana storage. This study provides an important theoretical basis for understanding the molecular mechanism of PA-mediated regulation of ripening and senescence of banana fruit, and the results may be helpful for improving banana storage technology.

\section{Materials and Methods}

Plant material and treatments. Banana fruit (AAA group, cv. Cavendish) fingers of the same shape and weight from the middle hands on the bunches with no visual defects were collected at the green mature stage ( $75 \%$ to $85 \%$ maturity) from a farm near Haikou, China. All the fruit fingers were cleaned and soaked in $0.1 \%(\mathrm{w} / \mathrm{v})$ fungicide (SporGon; Bayer, Leverkusen, Germany) for $1 \mathrm{~min}$ to eliminate potential microbes. The fruit were air-dried. Then, the selected banana fruit were randomly divided into two groups of 150 fingers each for the following treatments: $8 \mathrm{~min}$ in $10 \mathrm{~L}$ distilled water containing 0 (control) or $1 \%$ PA (Jianfeng, Tianjin, China) solution under reduced pressure of $\approx 0.05 \mathrm{MPa}$, as described by Chen et al. (2019). Both control and PA-treated fruit were subsequently treated with $100 \mu \mathrm{L} \cdot \mathrm{L}^{-1}$ ethylene for $24 \mathrm{~h}$, placed in 10 individual unsealed polyethylene plastic bags (thickness, $0.01 \mathrm{~mm}$ ), and stored at $25^{\circ} \mathrm{C}$ in the presence of $85 \%$ relative humidity for $7 \mathrm{~d}$. Samples were obtained after $0,1,3,5$, and $7 \mathrm{~d}$, the banana peel and pulp were collected separately. All samples were frozen in liquid nitrogen immediately after sampling and stored at $-80{ }^{\circ} \mathrm{C}$ for further use. All assessments were conducted in three biological replicates.

ENZYME ASSAYS. LAR and ANR activities were assayed following previously described protocols (Gagné et al., 2009). Powdered flesh samples $(1 \mathrm{~g})$ were extracted in $3 \mathrm{~mL}$ borate buffer ( $\mathrm{pH} 8.8$ ) containing $5 \mathrm{mmol} \cdot \mathrm{L}^{-1}$ vitamin $\mathrm{C}, 0.15 \%$ polyvinylpolypyrrolidone (PVPP), and $14 \mathrm{mmol} \cdot \mathrm{L}^{-1} \beta$-mercaptoethanol. The samples were vortexed before centrifuging at $12,000 g_{\mathrm{n}}$ for $20 \mathrm{~min}$ to obtain supernatant containing crude enzymes. The LAR assay mixture consisted of $0.5 \mathrm{~mL}$ crude LAR, $0.5 \mathrm{~mL} 0.1 \mathrm{~mol} \cdot \mathrm{L}^{-1}$ Tris-HCl buffer (pH 7.5), $0.5 \mathrm{~mL} 1.0$ $\mathrm{mol} \cdot \mathrm{L}^{-1}$ dihydroquercetin, and $0.5 \mathrm{~mL} 1.0 \mathrm{mmol} \cdot \mathrm{L}^{-1}$ reduced form of nicotinamide-adenine dinucleotide phosphate (NADPH). The mixture was incubated for $60 \mathrm{~min}$ at $37^{\circ} \mathrm{C}$, combined with $2 \mathrm{~mL} \mathrm{HCl} / \mathrm{methanol}(8 \%)$ and $2 \mathrm{~mL}$ vanillin/ methanol $(6 \%)$, and then incubated again for $60 \mathrm{~min}$ at $37{ }^{\circ} \mathrm{C}$. Absorbance was measured at $500 \mathrm{~nm}$ with a spectrophotometer (ultraviolet-1800; Shimadzu, Kyoto, Japan). We presented LAR activity as milligrams catechin per gram per hour. Powdered flesh samples $(1 \mathrm{~g})$ were extracted in $3 \mathrm{~mL}$ phosphate buffer (pH 7.4) with 1.0 g PVPP. Samples were vortexed before centrifuging at $12,000 g_{\mathrm{n}}$ for $20 \mathrm{~min}$ to obtain supernatant containing crude enzymes. The ANR mixture contained $0.4 \mathrm{~mL}$ crude ANR, $1.0 \mathrm{mmol} \cdot \mathrm{L}^{-1} \mathrm{NADPH}, 0.5 \mathrm{mmol} \cdot \mathrm{L}^{-1}$ vitamin $\mathrm{C}$, and $0.1 \mathrm{mmol} \cdot \mathrm{L}^{-1}$ phosphate buffer $(\mathrm{pH} 6.5)$. The mixture was incubated for $25 \mathrm{~min}$ at $45^{\circ} \mathrm{C}$. Absorbance was measured at 340 $\mathrm{nm}$ with a spectrophotometer (ultraviolet-1800). We presented ANR activity as milligrams epicatechin per gram per hour.

RNA SEQUENCING AND DATA ANALYSIS. Samples of peel from the control fruit (CF1) and the PA-treated fruit (PF1) on day 1 were selected for RNA sequencing. Three biological replicates were used. The mRNA was enriched using oligo dT beads and reverse-transcribed into cDNA using random primers. The cDNA was sequenced using the Illumina HiSeq 2500 (Illumina, San Diego, CA) instrument by Sangon Biotech Co. (Shanghai, China). Raw reads from each library were filtered separately. The adaptor sequences and reads with unknown sequences $(\mathrm{N})$ $>5 \%$ were removed. Furthermore, low-quality reads that contained more than $20 \% \mathrm{~N}$ [Phred quality score $(\mathrm{Q})<20$ bases] were discarded. After filtering, the quality of all clean reads was assessed using FastQC (Andrews, 2014). The reads were mapped to the banana reference genome, and gene expression levels were estimated with reads per kilobase per million mapped reads (Kim et al., 2013). DEGs between the different comparison groups were analyzed using DEGseq, and the statistical tests were revised for multiple testing using the Benjamini-Hochberg false discovery rate (FDR $<0.05$ ) (Wang et al., 2010). DEGs were selected based on fold change $\geq 2$ and adjusted to Q-value $\leq 0.05$. Gene ontology (GO) analyses were used to perform GO functional classification of DEGs and predict possible functions (Young et al., 2010). The Kyoto Encyclopedia of Genes and Genomes (KEGG) database was used to annotate the pathways involving DEGs with an E-value threshold of $10^{-5}$ (Kanehisa et al., 2008).

QUANTITATIVE REVERSE-TRANSCRIPTION POLYMERASE CHAIN REACTION (QRT-PCR). Total RNA was extracted using the RNA Plant Plus reagent following the manufacturer's instructions (DP437; Tiangen, Beijing, China). cDNA was synthesized using a PrimeScript RT reagent kit with gDNA eraser (Takara, Otsu, Japan). The PCR was performed with initial denaturation at $95^{\circ} \mathrm{C}$ for $3 \mathrm{~min}$, and then 40 cycles of $15 \mathrm{~s}$ at $95^{\circ} \mathrm{C}, 15 \mathrm{~s}$ at $55^{\circ} \mathrm{C}$, and $30 \mathrm{~s}$ at $72^{\circ} \mathrm{C}$. MaACT1 was selected as 
the reference gene (Chen et al., 2011). Primer 5.0 (version 5.0; PREMIER Biosoft., San Francisco, CA) was used for primer design. All primer sequences are listed in Supplemental Table 1.

Statistical analysis. Statistical analysis was conducted with statistical software (SPSS version 7.5; IBM Corp., Armonk, NY). Experiments were performed in a completely randomized manner with three replicates. All values are described as mean \pm standard error (SE). The least significant differences at the 5\% level were analyzed using DPS software (version 3.11; Zhejiang University, Hangzhou, China).

\section{Results}

ANR AND LAR ACTIVITIES. LAR and ANR are the last two principal enzymes that provide the cisflavan3-ol and transflavan-3-ol precursors needed for PA synthesis (Constabel, 2018). To study the effects of PA treatment on the activities of these two enzymes, we first measured the activities of these two enzymes. The results showed that both the peel and pulp of the banana fruit showed reduced ANR and LAR activities, but that PA treatment delayed the decrease in ANR and LAR activities; the activities of PA-treated fruit were always higher than those of the control fruit (Fig. 1). These results suggested that PA treatment might promote the accumulation of PA in banana fruit by affecting the ANR and LAR activities.

OVERVIEW OF SEQUENCING. To further investigate the regulation mechanism by which PA regulates banana fruit ripening and senescence, the CF1 and PF1 samples were used for transcriptome sequencing; 49,228,172 and 58,787,540 raw reads were generated from the CF1 and PF1 libraries, respectively. A summary of these sequencing results is presented in Table 1. After removing low-quality short sequences, 46,630,720 and 55,837,892 clean reads for CF1 and PF1, respectively, remained and were used for assembly. The Q20 percentages (sequencing error rate $<1 \%$ ) and guanine-cytosine (GC) percentages obtained from the CF1 and PF1 libraries were $98.07 \%$ and $54.61 \%$ and $98.02 \%$ and $54.44 \%$, respectively. These results suggested that the sequencing data had sufficient quantity and quality to ensure accurate sequence assembly and adequate transcriptome coverage. In addition, more than $92 \%$ of the clean reads that mapped to the banana genome comprised $\approx 90 \%$ uniquely mapped and $2 \%$ multiple-mapped clean reads. The uniquely mapped clean reads were used for analyzing gene expression levels, and results showed that 26,685 and 27,435 genes were expressed in CF1 and PF1, respectively.

Number ANd GO functional Classification OF DEGS. To screen DEGs, DESeq software was used; 1086 DEGs were identified between CF1 and PF1 samples based on the threshold q-value of 0.05 and $\log 2$-fold change of 2 , of which 872 DEGs
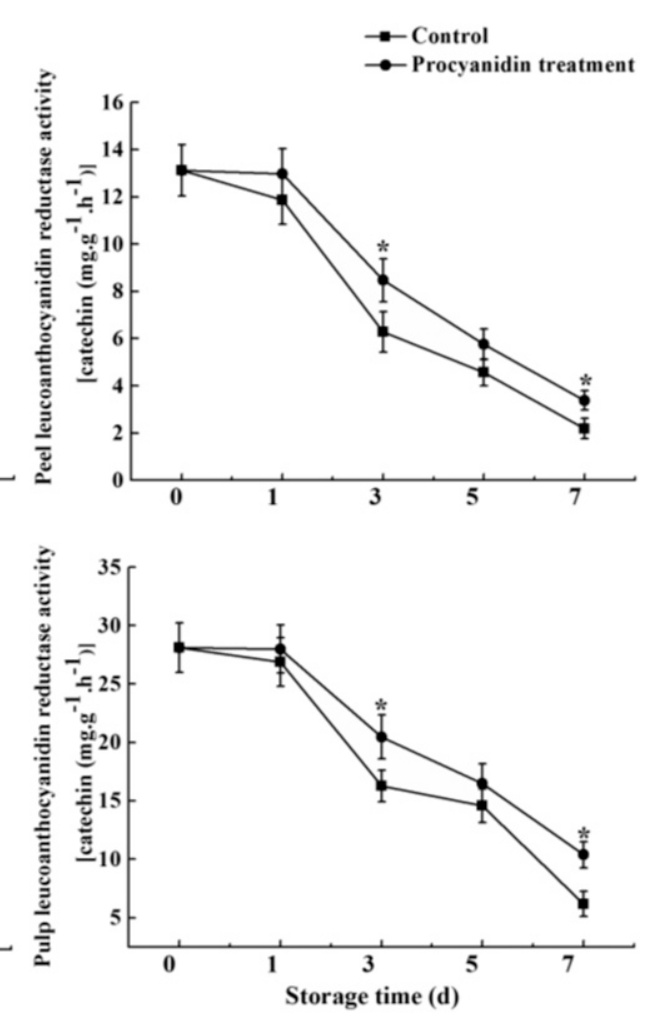

Effects of procyanidin treatment on the anthocyanidin reductase and leucoanthocyanidin reductase activities in the peel and pulp of banana fruit during $7 \mathrm{~d}$ of storage at $25^{\circ} \mathrm{C}$. Vertical bars represent the SE of three replicate assays. ${ }^{*} P<0.05$ compared with the control at the same time.

Table 1. Overview of the sequencing and assembly of the day 1 control fruit sample (CF1) and day 1 procyanidin-treated fruit sample (PF1).

\begin{tabular}{lcc}
\hline & CF1 & PF1 \\
\hline Total raw reads (no.) & $49,228,172$ & $58,787,540$ \\
Total clean reads (no.) & $46,630,720$ & $55,837,892$ \\
Total clean nucleotides (bp) & $6,637,406,199$ & $7,924,401,131$ \\
Phred quality score 20 (\%) & 98.07 & 98.02 \\
Guanine-cytosine (\%) & 54.61 & 54.44 \\
Uniquely mapped [no. (\%)] & $39,371,980(89.84)$ & $45,280,615(89.23)$ \\
Multiple mapped [no. (\%)] & $1,080,498(2.47)$ & $1,458,669(2.87)$ \\
Total expressed genes (no.) & 26,685 & 27,435 \\
\hline
\end{tabular}

were upregulated and 214 DEGs were downregulated (Fig. 2A). GOseq was used to analyze the distribution of DEGs in GO, which revealed the functions of the DEGs. Among the 1086 DEGs, 906 genes were successfully assigned to three categories (biological process, cellular component, and molecular function) with 50 groups (Fig. 2B). In the biological process category, the major DEGs were assigned to biological regulation, metabolic process, cellular process, and response to stimulus. Within the cellular component category, many DEGs were classified as cell, cell part, organelle, membrane, and membrane part. In the molecular function category, the subcategories of catalytic activity, transporter activity, and binding were mostly related (Fig. 2B).

KEGG Pathway analysis of DEGS. To analyze the biochemical pathways affected by PA, we mapped DEGs to the KEGG database for further analysis. A total of 206 DEGs 

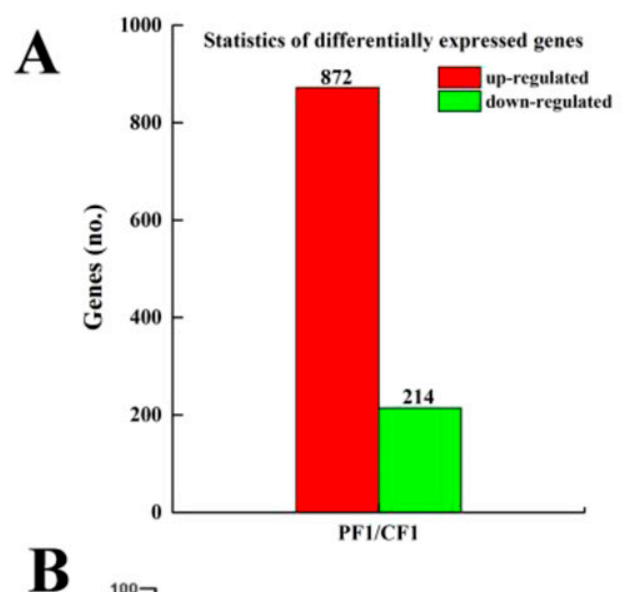

Gene function classification (GO)
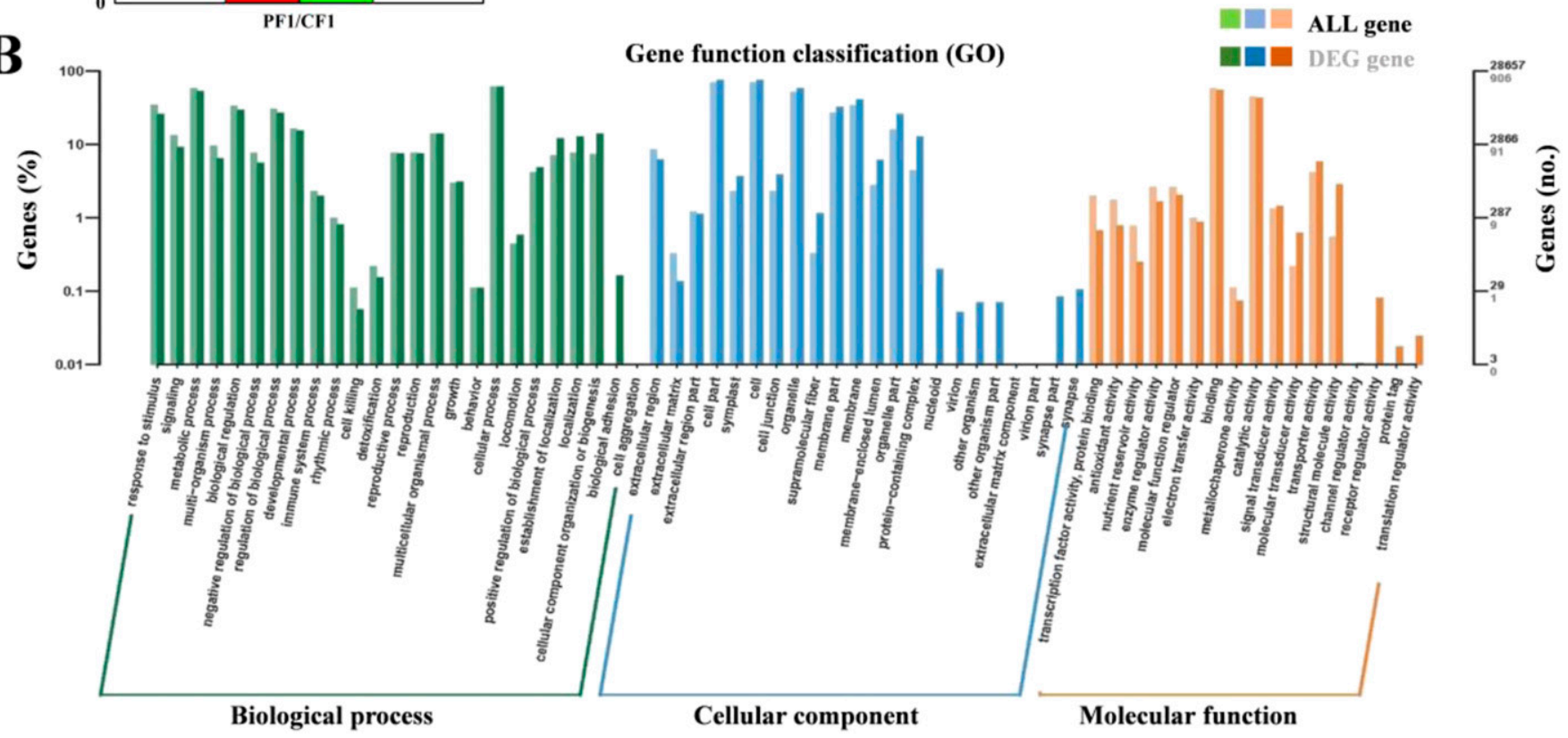

Fig. 2. Differentially expressed genes (DEGs) numbers and gene ontology (GO) functional classifications of banana peel samples of control fruit (CF1) and procyanidin-treated fruit (PF1) on day 1. (A) DEGs numbers between CF1 and PF1. (B) GO analysis of all genes and DEGs in banana. ALL gene indicates the gene number based on all banana genes. DEG gene indicates the gene number based on the DEGs of PF1 vs. CF1.

were assigned to $30 \mathrm{KEGG}$ pathways, the majority of which were related to metabolic pathways (Fig. 3A). Furthermore, PA treatment altered the KEGG pathway enrichment. As shown in Fig. 3B, the KEGG enrichment analysis revealed that the majority of the genes were involved in biosynthesis of amino acids, plant hormone signal transduction, MAPK signaling pathway, phenylalanine metabolism, and flavonoid biosynthesis. According to the results, the genes in the phenylalanine metabolism group and flavonoid biosynthesis group attracted our attention.

Validation of Candidate genes inVolved in PA BIOSYNTHESIS AND REGULATION. Based on GO and KEGG analyses, the genes encoding key biosynthetic enzymes and TFs controlling PA biosynthesis among the upregulated DEGs mainly from the biological regulation, metabolic process, response to stimulus, binding, phenylalanine metabolism, and flavonoid biosynthesis groups were screened. Finally, 6 genes encoding PA biosynthetic enzymes and 48 genes encoding WD40 (8), bHLH (15), and MYB (25) were found (Table 2). In addition, the expression levels of these genes in banana fruit during storage were detected using qRT-PCR. Among them, 21 genes ( 6 enzyme genes, 4 WD 40 genes, 5 bHLH genes, and 6
MYB genes) were induced by PA treatment during banana storage. The temporary names and gene identifications of these genes are shown in Supplemental Table 1. As shown in Fig. 4, during storage, the transcript levels of the biosynthetic enzymecoding genes in the control fruit changed little or decreased gradually. However, the expression levels of these genes in the PA-treated fruit increased in different degrees during 1 to $3 \mathrm{~d}$ of storage and then decreased gradually; the whole storage period was higher than that of the control fruit. Similarly, the results showed that the expression levels of the related WD40, MYB, and $b H L H$ regulators in the peel and pulp of banana fruit increased at different degrees after PA treatment during storage (Fig. 5). Among them, the MYB4, MYB5, bHLH4, bHLH5, WD401, and WD402 genes were significantly induced by PA treatment (Fig. 5). These results indicated that PA treatment induced the expression of PA synthesis-related genes, which was conducive to the accumulation of PA in banana fruit.

\section{Discussion}

As a class of flavonoids, PA possesses antiaging and antioxidant properties; furthermore, it has been shown to 
A

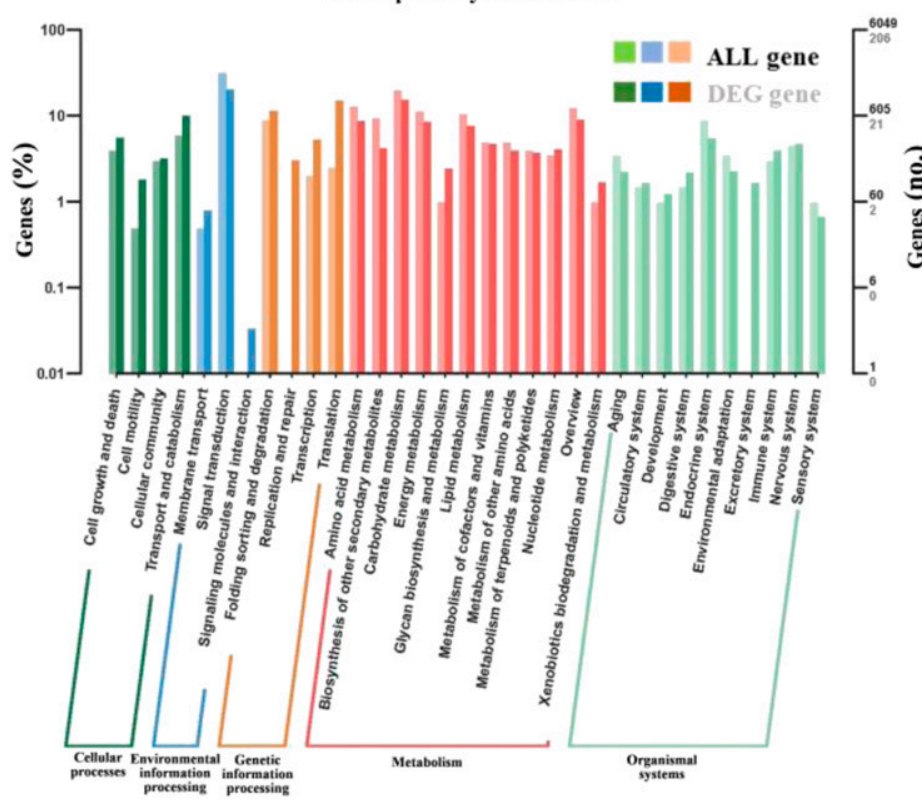

B

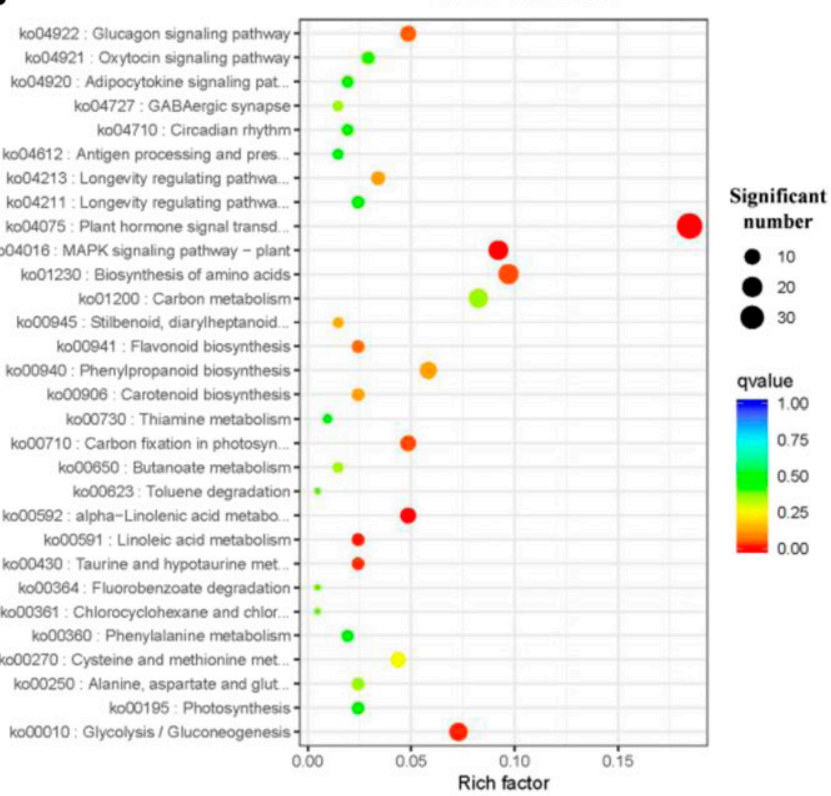

Fig. 3. The Kyoto Encyclopedia of Genes and Genomes (KEGG) pathway analysis of banana peel samples of control fruit (CF1) and procyanidin-treated fruit (PF1) on day 1. (A) The KEGG pathway classification of all genes and differentially expressed genes (DEGs) in banana. (B) The KEGG pathway enrichment of DEGs. ALL gene indicates the gene number based on all banana genes. DEG gene indicates the gene number based on the DEGs of PF1 vs. CF1.

Table 2. Numbers and $\log 2$ ratios of the genes involved in procyanidin biosynthesis in the differentially expressed genes of banana fruit transcriptome.

\begin{tabular}{|c|c|c|}
\hline $\begin{array}{l}\text { Gene } \\
\text { family }\end{array}$ & $\begin{array}{c}\text { Annotated } \\
\text { sequences (no.) }\end{array}$ & Gene (log2 ratio) \\
\hline DFR & 1 & Ma04_g10620 (2.51) \\
\hline LAR & 1 & Ma05_g16480 (2.63) \\
\hline ANR & 1 & Ma08_g01380 (2.11) \\
\hline WD40 & 8 & $\begin{array}{l}\text { Ma00_g04180 (2.42), Ma01_g05340 (8.22), Ma01_g05340 (1.89), Ma00_g00980 (2.16), Ma01_g04190 (15.77), } \\
\text { Ma01_g04720 (3.12), Ma01_g08850 (2.47), Ma01_g10940 (2.88) }\end{array}$ \\
\hline MYB & 25 & $\begin{array}{l}\text { Ma01_g02850 (4.62), Ma02_g05880 (2.09), Ma03_g29510 (3.11), Ma04_g28510 (1.96), Ma04_g31800 (4.01), } \\
\text { Ma04_g34660 (2.34), Ma05_g03690 (2.13), Ma05_g08960 (2.62), Ma05_g25680 (1.95), Ma05_g30120 } \\
\text { (2.93), Ma06_g00910 (2.09), Ma06_g14470 (2.19), Ma06_g33100 (2.34), Ma07_g02470 (2.63), } \\
\text { Ma07_g08110 (2.55), Ma07_g17600 (3.12), Ma07_g22540 (3.56), Ma08_g01300 (2.74), Ma08_g32760 } \\
\text { (2.22), Ma09_g04930 (5.13), Ma09_g23100 (1.98), Ma09_g24640 (2.45), Ma11_g03860 (2.66), } \\
\text { Ma11_g04680 (3.33), Ma00_g01590 (3.23) }\end{array}$ \\
\hline
\end{tabular}

improve fruit quality and delay senescence in banana during storage and inhibit the decrease of in vivo PA content (Chen et al., 2019). Hence, in this study, we aimed to determine the mechanism of PA treatment regulating PA accumulation in banana fruit during storage. PAs are biosynthesized through the phenylpropanoid and flavonoid pathways containing a variety of enzymes (He et al., 2008). LAR and ANR are the most important enzymes of PA biosynthesis; the formation of flavan-3-ols [2,3-cis-(-)-flavan-3-ols and 2,3-trans-(+)-flavan3-ols] is achieved by LAR and ANR (Bogs et al., 2005). LAR and ANR activities have been observed in several plants, and their activities are usually positively correlated with the accumulation of PA (Cantin et al., 2009; Hassanpour, 2015; Marles et al., 2003). In the present study, the activities of ANR and LAR in PA-treated banana fruit were higher than those in the control $(P<0.05)$ (Fig. 1), indicating that PA treatment 


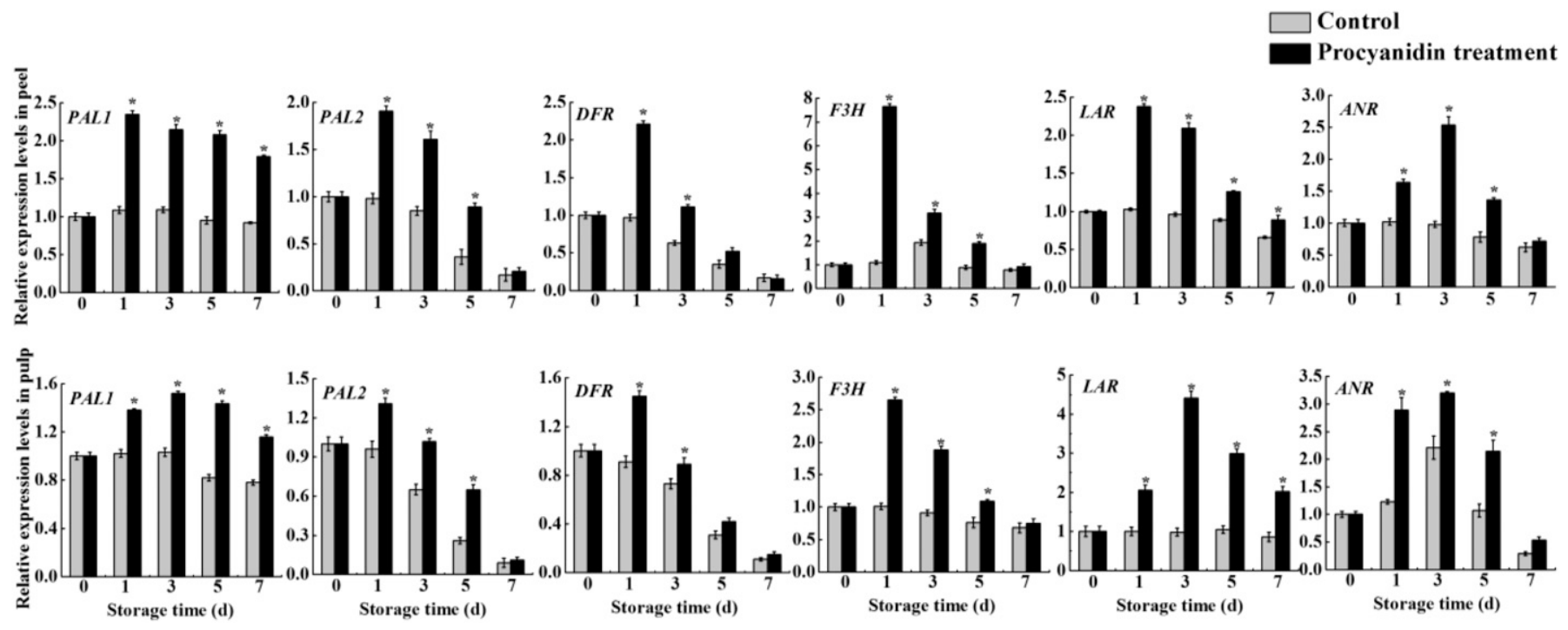

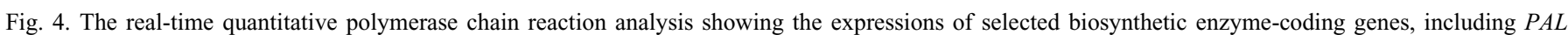
(Ma09_g15390), PAL (Ma02_g00760), DFR (Ma04_g10620), F3H (Ma06_g17160), LAR (Ma05_g16480), and ANR (Ma08_g01380), in the peel and pulp of banana fruit treated with or without procyanidin during $7 \mathrm{~d}$ of storage at $25^{\circ} \mathrm{C}$. The expression levels at different time points were expressed as ratios relative to the harvest time (day 0 for the control), which was set at 1 . Vertical bars represent the SE of three replicate assays. $* P<0.05$ compared with the control at the same time.

triggers the key enzymes of the PA biosynthetic pathway in banana fruit. More importantly, the biosynthesis of PA is regulated primarily at the gene expression level (Constabel, 2018). Therefore, the expression levels of the PA biosynthetic and regulatory genes are important.

RNA-seq analysis is an important tool for obtaining information regarding genes involved in the physiological and metabolic processes associated with fruit development and ripening in nonmodel fruit species (Travisany et al., 2019). In this study, cDNA libraries from PA-treated and control fruit were constructed and sequenced. As a result, $1086 \mathrm{DEGs}$ were identified in PA-treated and control samples (Fig. 2A). Six genes encoding PA biosynthetic enzymes and 48 genes encoding WD40 (8 genes), bHLH (15 genes) and MYB (25 genes) were found during the RNA-seq analysis (Table 2). The main biosynthetic genes encoding enzymes involved in flavonoid and PA biosynthetic pathways have been studied in many species. For instance, the expression of salicylic acidinduced $P A L$ triggered flavonoid accumulation in broccoli [Brassica oleracea var. italica (Pérez-Balibrea et al., 2011)]. Exogenous melatonin pretreatment improved anthocyanin accumulation by regulating $F 3 H$ and $D F R$ gene expression in cabbage [Brassica pekinensis (Zhang et al., 2016)]. Manipulation of $D F R$ was successfully performed in rose (Rosa rugosa) petals to generate blue hues with anthocyanin accumulation (Dixon et al., 2013). Moreover, Ghag et al. (2015) reported that silencing of MusaANRI reduced the PA content in transgenic banana plants (Ghag et al., 2015). Ectopic expression of $L A R$ genes from Medicago or cacao (Theobroma cacao) and $A N R$ genes from apple or grape (Vitis vinifera) in tobacco (Nicotiana tabacum) petals resulted in the accumulation of PA (Bogs et al., 2005; Han et al., 2012; Liu et al., 2013; Pang et al., 2007). In the present study, the transcript levels of the PA biosynthetic enzyme genes PAL1, PAL2, $F 3 H, D F R, A N R$, and $L A R$ in the PA-treated fruit were higher than those in the control fruit during storage (Fig. 4), which may cause the accumulation of PA in banana fruit and is consistent with the results of our previous studies (Chen et al., 2019). Moreover, the flavonoid and PA biosynthetic pathways are regulated by different MBW complexes (Feller et al., 2011; Zhang et al., 2018). The A. thaliana MYB123 protein interacted with specific bHLH (TT8) and WD40 (TTG1) proteins to determine PA synthesis in the seedcoat (Xu et al., 2015). In grape, the bHLH partner of the PA-MBW complex, VvMYC1, can interact with the PA-specific VvMYBPA1 to form a functional complex for the PA synthesis pathway (Hichri et al., 2010). In apple, MdMYB11 activated flavonoid structural genes through the MBW ternary complex, which promoted anthocyanin and PA biosynthesis (An et al., 2015). Wang et al. (2017) found that MdMYB12 interacted with MdbHLH3 and MdbHLH33 to regulate PA synthesis. Many WD40, MYB, and bHLH TFs have been shown to form a functional complex and regulate flavonoid and PA biosynthesis by acting on promoters of biosynthetic enzyme genes (Liu et al., 2018; Mehrtens et al., 2005; Stracke et al., 2007). In the present study, PA treatment upregulated the expression of MBW-related TFs, including four $W D 40$, five $b H L H$, and six $M Y B$ genes (Fig. 5). Therefore, whether and how the WD40, MYB, and bHLH TFs identified in this study regulate the biosynthetic enzyme-coding genes should be investigated in the future.

In summary, in this study, the activities of key PA synthesis enzymes such as ANR and LAR were higher in PA-treated banana fruit than those in the control fruit during storage. The PA treatment also induced the expression of the structural and regulatory genes involved in PA biosynthesis, leading to higher transcript levels of these genes in PA-treated banana fruit during storage. These results may induce the accumulation of PA in banana fruit and provide a theoretical basis for the effects of PA on banana senescence. 

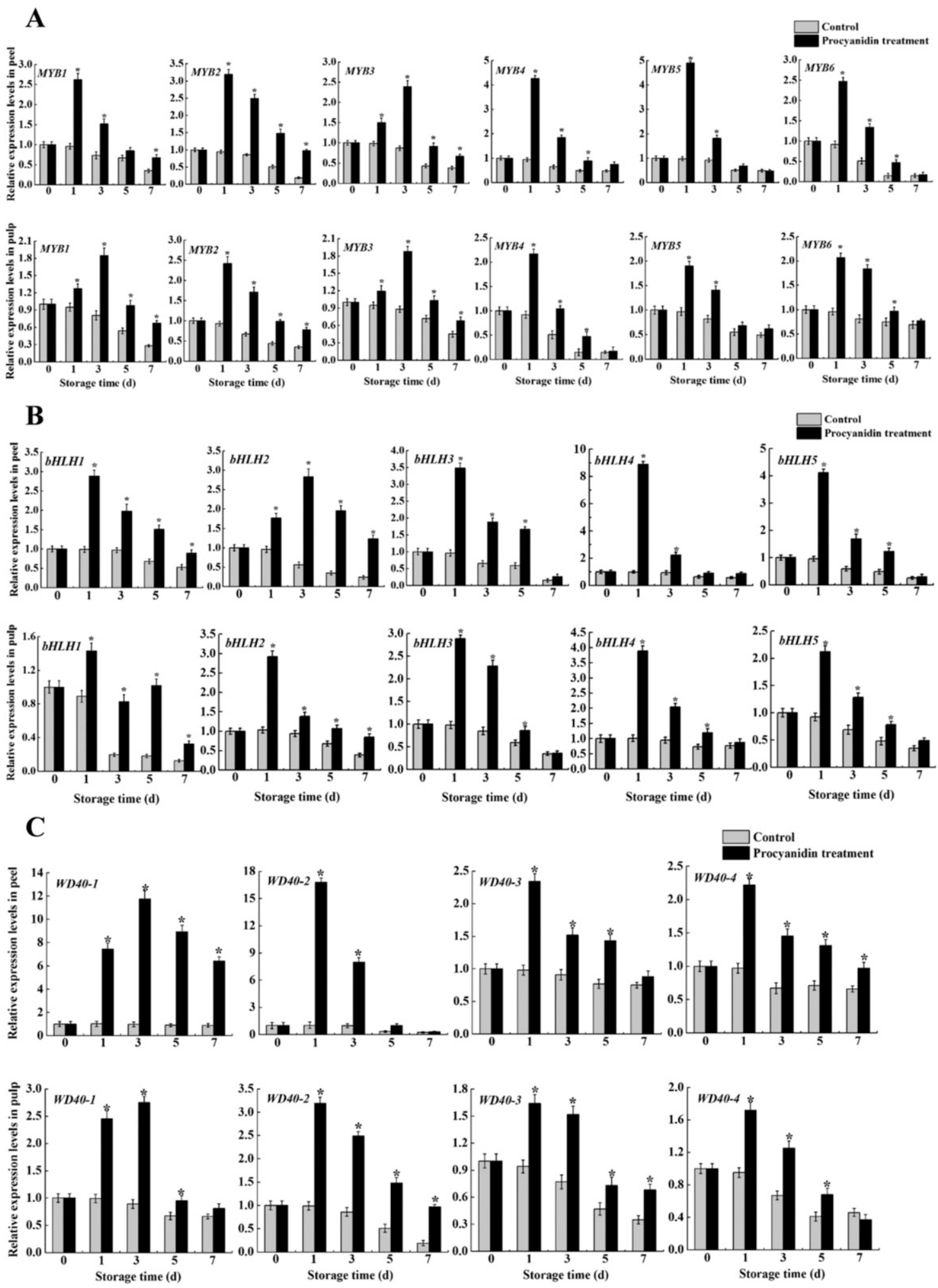

Fig. 5. The real-time quantitative polymerase chain reaction analysis showing the expression of selected transcription factors in the peel and pulp of banana fruit treated with or without procyanidin during $7 \mathrm{~d}$ of storage at $25^{\circ} \mathrm{C}$. (A) Expressions of MYB1 (Ma00_g01590), MYB2 (Ma07_g22540), MYB3 (Ma09_g23100), MYB4 (Ma01_g02850), MYB5 (Ma09_g04930), and MYB6 (Ma11_g03860). (B) Expressions of bHLH1 (Ma03_g18060), bHLH2 (Ma07_g02820), bHLH3 (Ma01_g12390), bHLH4 (Ma07_g04810), and bHLH5 (Ma11_g16400). (C) Expressions of WD40-1 (Ma01_g05340), WD40-2 (Ma01_g04190), WD40-3 (Ma01_g04720), and WD40-4 (Ma01_g10940). The expression levels at different time points were expressed as ratios relative to the harvest time (day 0 for the control), which was set at 1 . Vertical bars represent the SE of three replicate assays. ${ }^{*} P<0.05$ compared with the control at the same time. 


\section{Literature Cited}

An, X.H., Y. Tian, K.Q. Chen, X.J. Liu, D.D. Liu, X.B. Xie, C.G. Cheng, P.H. Cong, and Y.J. Hao. 2015. MdMYB9 and MdMYB11 are involved in the regulation of the JA-induced biosynthesis of anthocyanin and proanthocyanidin in apples. Plant Cell Physiol. 56:650-662, doi: 10.1093/pcp/pcu205.

Andrews, S. 2014. FastQC: A quality control tool for high throughput sequence data. 21 Dec. 2020. <http://www.bioinformatics.babraham.ac.uk/projects/fastqc/>.

Bogs, J., M.O. Downey, J.S. Harvey, A.R. Ashton, G.J. Tanner, and S.P. Robinson. 2005. Proanthocyanidin synthesis and expression of genes encoding leucoanthocyanidin reductase and anthocyanidin reductase in developing grape berries and grapevine leaves. Plant Physiol. 139:652-663, doi: 10.1104/pp.105.064238.

Cantin, C.M., M.A. Moreno, and Y. Gogorcena. 2009. Evaluation of the antioxidant capacity, phenolic compounds, and vitamin $\mathrm{C}$ content of different peach and nectarine [Prunus persica (L.) Batsch] breeding progenies. J. Agr. Food Chem. 57:4586-4592, doi: 10.1021/jf900385a.

Chen, J., F.F. Li, Y.X. Li, Y.S. Wang, C.Z. Wang, D.B. Yuan, and Y.M. Jiang. 2019. Exogenous procyanidin treatment delays senescence of harvested banana fruit by enhancing antioxidant responses and in vivo procyanidin content. Postharvest Biol. Technol. 158:110999, doi: 10.1016/j.postharvbio.2019.110999.

Chen, L., H.Y. Zhong, J.Y. Kuang, J.G. Li, W.J. Lu, and J.Y. Chen. 2011. Validation of reference genes for RT-qPCR studies of gene expression in banana fruit under different experimental conditions. Planta 234:377-390, doi: 10.1007/s00425-011-1410-3.

Chen, W., Z. Zhang, Y. Shen, X. Duan, and Y. Jiang. 2014. Effect of tea polyphenols on lipid peroxidation and antioxidant activity of litchi (Litchi chinensis Sonn.) fruit during cold storage. Molecules 19:16837-16850, doi: 10.3390/molecules191016837.

Chibane, L.B., P. Degraeve, and H. Ferhout. 2019. Plant antimicrobial polyphenols as potential natural food preservatives. J. Sci. Food Agr. 99:1457-1474, doi: 10.1002/jsfa.9357.

Constabel, C.P. 2018. Molecular controls of proanthocyanidin synthesis and structure: Prospects for genetic engineering in crop plants. J. Agr. Food Chem. 66:9882-9888, doi: 10.1021/acs.jafc.8b02950.

Deluc, L., F. Barrieu, C. Marchive, V. Lauvergeat, A. Decendit, T. Richard, and S. Hamdi. 2006. Characterization of a grapevine R2R3MYB transcription factor that regulates the phenylpropanoid pathway. Plant Physiol. 140:499-511, doi: 10.1104/pp.105.067231.

Dixon, R.A., C.G. Liu, and J.H. Jun. 2013. Metabolic engineering of anthocyanins and condensed tannins in plants. Curr. Opin. Biotechnol. 24:329-335, doi: 10.1016/j.copbio.2012.07.004.

Elitzur, T., E. Yakir, L. Quansah, F. Zhangjun, J.T. Vrebalov, E. Khayat, J.J. Giovannoni, and H. Friedman. 2016. Banana MaMADS transcription factors are necessary for fruit ripening and molecular tools to promote shelf-life and food security. Plant Physiol. 171:380, doi: 10.1104/pp.15.01866.

Feller, A., K. Machemer, E.L. Braun, and E. Grotewold. 2011. Evolutionary and comparative analysis of MYB and bHLH plant transcription factors. Plant J. 66:94-116, doi: 10.1111/j.1365313X.2010.04459.x.

Gagné, S., S. Lacampagne, O. Claisse, and L. Gény. 2009. Leucoanthocyanidin reductase and anthocyanidin reductase gene expression and activity in flowers, youngberries and skins of Vitis vinifera L.cv. Cabernet-Sauvignon during development. Plant Physiol. Biochem. 47:282-290, doi: 10.1016/j.plaphy.2008.12.004.

Ghag, S.B., U.K.S. Shekhawat, and T.R. Ganapathi. 2015. Silencing of MusaANR1 gene reduces proanthocyanidin content in transgenic banana plants. Plant Cell Tissue Organ Cult. 121:693-702, doi: 10.1007/s11240-015-0739-x.

Han, Y., S. Vimolmangkang, R.E. Soria-Guerra, and S.S. Korban. 2012. Introduction of apple ANR genes into tobacco inhibits expression of both CHI and DFR genes in flowers, leading to loss of anthocyanin. J. Expt. Bot. 63:2437-2447, doi: 10.1093/jxb/err415.
Hassanpour, H. 2015. Effect of Aloe vera gel coating on antioxidant capacity, antioxidant enzyme activities and decay in raspberry fruit. Lebensm. Wiss. Technol. 60:495-501, doi: 10.1016/j.1wt. 2014.07.049.

He, F., Q.H. Pan, Y. Shi, and C.Q. Duan. 2008. Biosynthesis and genetic regulation of proanthocyanidins in plants. Molecules 13:2674-2703, doi: 10.3390/molecules13102674.

Hichri, I., S.C. Heppel, J. Pillet, C. Leon, S. Czemmel, S. Delrot, V. Lauvergeat, and J. Bogs. 2010. The basic helix-loop-helix transcription factor MYC1 is involved in the regulation of the flavonoid biosynthesis pathway in grapevine. Mol. Plant 3:509-523, doi: 10.1093/mp/ssp118.

Jing, Y.J., J.H. Huang, and X.Q. Yu. 2019. Maintenance of the antioxidant capacity of fresh-cut pineapple by procyanidin-grafted chitosan. Postharvest Biol. Technol. 154:79-86, doi: 10.1016/j.postharvbio.2019.04.022.

Kanehisa, M., M. Araki, S. Goto, M. Hattori, M. Hirakawa, M. Itoh, and Y. Yamanishi. 2008. KEGG for linking genomes to life and the environment. Nucleic Acids Res. 36:D480-D484, doi: 10.1093/nar/ gkm882.

Kim, D., G. Pertea, C. Trapnell, H. Pimentel, R. Kelley, and S.L. Salzberg. 2013. TopHat2: Accurate alignment of transcriptomes in the presence of insertions, deletions and gene fusions. Genome Biol. 14:R36, doi: 10.1186/gb-2013-14-4-r36.

Liu, Y.J., H. Hou, X.L. Jiang, P.Q. Wang, X.L. Dai, W. Chen, L.P. Gao, and T. Xia. 2018. A WD40 repeat protein from Camellia sinensis regulates anthocyanin and proanthocyanidin accumulation through the formation of MYB-bHLH-WD40 ternary complexes. Intl. J. Mol. Sci. 19:1686, doi: 10.3390/ijms19061686.

Liu, Y., Z. Shi, S. Maximova, M.J. Payne, and M.J. Guiltinan. 2013. Proanthocyanidin synthesis in Theobroma cacao: Genes encoding anthocyanidin synthase, anthocyanidin reductase, and leucoanthocyanidin reductase. BMC Plant Biol. 13:202, doi: 10.1186/14712229-13-202.

Marles, M.A., H. Ray, and M.Y. Gruber. 2003. New perspectives on proanthocyanidin biochemistry and molecular regulation. Phytochemistry 64:367-383, doi: 10.1016/s0031-9422(03)00377-7.

Mehrtens, F., H. Kranz, P. Bednarek, and B. Weisshaar. 2005. The arabidopsis transcription factor MYB12 is a flavonol-specific regulator of phenylpropanoid biosynthesis. Plant Physiol. 138:10831096, doi: 10.1104/pp.104.058032.

Pang, Y., G.J. Peel, E. Wright, Z. Wang, and R.A. Dixon. 2007. Early steps in proanthocyanidin biosynthesis in the model legume Medicago truncatula. Plant Physiol. 145:601-615, doi: 10.1104/ pp.107.107326.

Pérez-Balibrea, S., D.A. Moreno, and C. García-Viguera. 2011. Genotypic effects on the phytochemical quality of seeds and sprouts from commercial broccoli cultivars. Food Chem. 125:348-354, doi: 10.1016/j.foodchem.2010.09.004.

Stracke, R., H. Ishihara, G. Huep, A. Barsch, F. Mehrtens, K. Niehaus, and B. Weisshaar. 2007. Differential regulation of closely related R2R3-MYB transcription factors controls flavonol accumulation in different parts of the Arabidopsis thaliana seedling. Plant J. 50:660677, doi: 10.1111/j.1365-313X.2007.03078.x.

Shi, M.Z. and D.Y. Xie. 2014. Biosynthesis and metabolic engineering of anthocyanins in Arabidopsis thaliana. Recent Pat. Biotechnol. 8:47-60, doi: 10.2174/187220830766613121 8123538 .

Travisany, D., A. Ayala-Raso, A.D. Genova, L. Monsalve, M. Bernales, J.P. Martínez, M. González-Agüero, B. Defilippi, S. Cherian, A. Maass, and L. Fuentes. 2019. RNA-Seq analysis and transcriptome assembly of raspberry fruit (Rubus idaeus "Heritage") revealed several candidate genes involved in fruit development and ripening. Scientia Hort. 254:26-34, doi: 10.1016/ j.scienta.2019.04.018.

Wang, L., Z. Feng, X. Wang, X. Wang, and X. Zhang. 2010. DEGseq: An $\mathrm{R}$ package for identifying differentially expressed genes from RNAseq data. Bioinformatics 26:136-138, doi: 10.1093/ bioinformatics/btp612. 
Wang, N., H. Xu, S. Jiang, Z. Zhang, N. Lu, H. Qiu, C. Qu, Y. Wang, S. Wu, and X. Chen. 2017. MYB12 and MYB22 play essential roles in proanthocyanidin and flavonol synthesis in red-fleshed apple (Malus sieversii f. niedzwetzkyana). Plant J. 90:276-292, doi: 10.1111/tpj.13487.

Winkel-Shirley, B. 2001. Flavonoid biosynthesis. A colorful model for genetics, biochemistry, cell biology, and biotechnology. Plant Physiol. 126:485-493, doi: 10.1104/pp.126.2.485.

Xi, Y., X.G. Fan, H.D. Zhao, X.H. Li, J.K. Cao, and W.B. Jiang. 2017. Postharvest fruit quality and antioxidants of nectarine fruit as influenced by chlorogenic acid. Lebensm. Wiss. Technol. 75:537544, doi: 10.1016/j.lwt.2016.10.004.

Xiao, Y., J.L. Dong, Z.T. Yin, Q.G. Wu, Y.M. Zhou, and X.L. Zhou. 2018. Procyanidin B2 protects against D-galactose-induced mimetic aging in mice: Metabolites and microbiome analysis. Food Chem. Toxicol. 119:141-149, doi: 10.1016/j.fct.2018.05.017.

$\mathrm{Xu}$, W., D. Grain, S. Bobet, G.J. Le, J. Thévenin, Z. Kelemen, L. Lepiniec, and C. Dubos. 2014. Complexity and robustness of the flavonoid transcriptional regulatory network revealed by comprehensive analyses of MYB-bHLH-WDR complexes and their targets in arabidopsis seed. New Phytol. 202:132-144, doi: 10.1111/nph.12620.

Xu, W.J., C. Dubos, and L. Lepiniec. 2015. Transcriptional control of flavonoid biosynthesis by MYB-bHLH-WDR complexes. Trends Plant Sci. 20:176-185, doi: 10.1016/j.tplants.2014.12.001.

Yang, J.F., W.J. Shi, B.B. Li, Y.C. Bai, and Z.X. Hou. 2019. Preharvest and postharvest UV radiation affected flavonoid metabolism and antioxidant capacity differently in developing blueberries (Vaccinium corymbosum L.). Food Chem. 301:125248, doi: 10.1016/j.foodchem. 2019.125248

Young, M.D., M.J. Wakefield, G.K. Smyth, and A. Oshlack. 2010. Gene ontology analysis for RNA-seq: Accounting for selection bias. Genome Biol. 11:R14, doi: 10.1186/gb-2010-11-2-r14.

Yun, Z., T.T. Li, H.J. Gao, H. Zhu, V.P. Gupta, Y.M. Jiang, and X.W. Duan. 2019. Integrated transcriptomic, proteomic, and metabolomics analysis reveals peel ripening of harvested banana under natural condition. Biomolecules 9:167, doi: 10.3390/biom9050167.

Zhang, J., H.F. Xu, N. Wang, S.H. Jiang, H.C. Fang, Z.Y. Zhang, G.X. Yang, Y.C. Wang, Y.S. Meng, L. Xu, and X.S. Chen. 2018. The ethylene response factor MdERF1B regulates anthocyanin and proanthocyanidin biosynthesis in apple. Plant Mol. Biol. 98:205218, doi: 10.1007/s11103-018-0770-5.

Zhang, N., Q. Sun, H. Li, X. Li, Y. Cao, H. Zhang, S. Li, L. Zhang, Y. Qi, S. Ren, B. Zhao, and Y.D. Guo. 2016. Melatonin improved anthocyanin accumulation by regulating gene expressions and resulted in high reactive oxygen species scavenging capacity in cabbage. Front Plant Sci. 7:197, doi: 10.3389/fpls.2016.00197.

Zhang, Z.K., D.J. Huber, H.X. Qu, Z. Yun, H. Wang, Z.H. Huang, H. Huang, and Y.M. Jiang. 2015. Enzymatic browning and antioxidant activities in harvested litchi fruit as influenced by apple polyphenols. Food Chem. 171:191-199, doi: 10.1016/j.foodchem. 2014.09.001. 
Supplemental Table 1. Names, gene identifications (ID), and primers of the selected genes used for real-time quantitative polymerase chain reaction analysis

\begin{tabular}{|c|c|c|c|c|}
\hline Gene name & Gene & Forward primer $\left(5^{\prime}-3^{\prime}\right)$ & Reverse primer $\left(5^{\prime}-3^{\prime}\right)$ & Product (bp) \\
\hline$P A L 1$ & Ma09_g15390 & CGTTGACGAAGCCGAAGC & CGTGCGGATCACCTCTATTT & 83 \\
\hline PAL2 & Ma02_g00760 & CAACGGTGAGAAGGAGAAGG & GGGTACGATCTGCATTCCTTG & 168 \\
\hline$D F R$ & Ma04_g10620 & CAGAATGGATACATCGTCAGGG & ААСАСТССТТСАСАТСССТТG & 167 \\
\hline$F 3 H$ & Ma06_g17160 & СТCССCTTGTGCCTGATG & TAGAAGTTGACCCTCATGCAG & 200 \\
\hline$L A R$ & Ma05_g16480 & ACAGTCGCACCGTGAATAGA & AACAACGCTGGCTGGAAT & 176 \\
\hline$A N R$ & Ma08_g01380 & GCACTTTCTGCGTCGTAT & TGACAGCGTAGCCCTTCT & 186 \\
\hline$M Y B 1$ & Ma00_g01590 & CCTATAAGCAAGTCCATCCCG & ATGATTTCGTCCTCTTCCGC & 119 \\
\hline MYB2 & Ma07_g22540 & СTTTTCCAACGCCAGATCAAG & GTTTCGTCAAAGAGCCATGTC & 80 \\
\hline$M Y B 3$ & Ma09_g23100 & ATTTACCGTCCAATCCCCAG & GTAGGAAGATGGTGACGCAG & 135 \\
\hline MYB4 & Ma01_g02850 & GACAACGAGATTAAGAACCACTG & TGTAATGTTAGATGGCGGACG & 114 \\
\hline MYB5 & Ma09_g04930 & AAGATGTGTCGGGAGGTTTG & AGGTCATTGCTCATGTGGTAG & 147 \\
\hline MYB6 & Ma11_g03860 & GATCTTGGTCGACTACATCCAG & ATGGCGTGGAGTTGGATG & 183 \\
\hline$b H L H 1$ & Ma03_g18060 & AGAAGGACAGGATGGGAGAG & CCTCCGAAAGAACAGATGCT & 90 \\
\hline bHLH2 & Ma07_g02820 & ATCCGATATCTGCGAGCAAC & AАTCCTCTTCATCTGCCACC & 116 \\
\hline bHLH3 & Ma01_g12390 & TCCTAGCTTGCCTCATTTCC & TTGTCACCAGTCATACTCAGC & 195 \\
\hline bHLH4 & Ma07_g04810 & GCAAGGTCAGATTACTCTTCGG & GAGGTTAGGGTTTTCGGTGAG & 197 \\
\hline bHLH5 & Ma11_g16400 & TGCGGTCTCATACATCAACG & СТТСАСТТССАТСТССАССС & 178 \\
\hline WD40-1 & Ma01_g05340 & CGTCTAGCTACCTCGTCATTTG & TTTCTCCATCGCCATCACAG & 163 \\
\hline WD40-2 & Ma01_g04190 & TGAAGGGAAACTCTTGGGATG & AAGAGCATAGGGAGCAATGAG & 88 \\
\hline WD40-3 & Ma01_g04720 & GAAGGACAATCGACACCAGAG & CAGCTATACCTAACCCAATCGG & 196 \\
\hline WD40-4 & Ma01_g10940 & TTGACCTAGCATCCAAGTGTG & AAGCCACAGAATCCAGATCG & 147 \\
\hline$M a A C T 1$ & HQ853237 & TGGTATGGAAGCCGCTGGTA & TCTGCTGGAATGTGCTGAGG & 236 \\
\hline
\end{tabular}

